\title{
Surface molecular alignment by in-plane anchoring in the cell showing the V-shaped switching
}

\author{
S. S. Seomun and J. K. Vij ${ }^{\text {a) }}$ \\ Department of Electronic Engineering, Trinity College, University of Dublin, Dublin 2, Ireland
}

N. Hayashi and T. Kato

Institute for Molecular Science, Myodaiji, Okazaki-shi, Japan

\author{
A. Fukuda \\ Department of Kansei Engineering, Shinshu University, Ueda-shi, Nagano, Japan
}

(Received 21 March 2001; accepted for publication 15 June 2001)

\begin{abstract}
The surface molecular alignment in a sandwich cell showing V-shaped switching has been investigated using a photocontrolling method. The results indicate that the molecules on the rubbed surface are aligned parallel to the rubbing direction as in an open cell with an air-liquid crystal boundary. This alignment is due to strong in-plane anchoring, due to which the small twisted state appears along the cell thickness through intralayer molecular interaction. (C) 2001 American Institute of Physics. [DOI: 10.1063/1.1389770]
\end{abstract}

The study of V-shaped switching [Fig. 1(a)] is one of the major areas of current interest in the field of chiral smectic liquid crystals. ${ }^{1-8}$ The reason for interest in them lies in their promise of realizing new types of liquid crystal displays (LCDs). ${ }^{9}$ Since the discovery of V-shaped switching, random $^{1,2}$ and the collective models ${ }^{5,6}$ were proposed to explain its switching mechanism. Many physical properties have been investigated which support one or both these models. However, the nature of the liquid crystal phase and the molecular orientation remain problems for realizing $\mathrm{V}$-shaped switching. Understanding the surface-molecule interactions is important for analyzing the V-shaped switching phenomenon. The liquid crystal samples including the Inui mixture have a helix with a finite pitch in freestanding films. ${ }^{3,4}$ Meanwhile, the V-shaped switching cell in which the helix is suppressed, has an average optical axis parallel to the smectic layer normal $l$ for an applied voltage of $V=0$. This has been confirmed by good extinction under crossed polarizers when one of them is along $l . .^{3,5,6}$ Recently, Clark et al. suggested the electrostatic model to explain the stabilization of molecules like this. ${ }^{7}$ According to their model, polarization charge self-interaction becomes important with material having sufficiently large spontaneous polarization $P$. This makes $P$ in the bulk align uniformly parallel to the aligning surface $(\varphi=\pi / 2)$ through $P$ on both surfaces heads for the surface normal $(\varphi=0$ and $\pi)$. In addition, it was also suggested that the molecules in the entire cell thickness may also align uniformly with $\varphi=\pi / 2$.

On the contrary, we observed the surface molecular alignment of the Inui mixture using an open cell with an air-liquid crystal boundary. ${ }^{8} \operatorname{In} \operatorname{Sm} A$ phase, the smectic layer normal $l$ deviates at an angle of $\sim 12^{\circ}$ from the rubbing axis $r$, rather similar to the behavior in a sandwich cell. However, in $\operatorname{Sm} X^{*}$ phase, the director $n$ becomes parallel to $r$ in an open cell. Considering an apparent tilt angle of $\sim 35^{\circ}$, it is very clear that the direction of $P$ on the surface is closer to

\footnotetext{
a) Author to whom correspondence should be addressed; electronic mail: jvij@tcd.ie
}

$\varphi=\pi / 2$ than to 0 or $\pi$, contrary to Clark et al.'s early predictions. $^{7,8}$ And, the small twisted state along the cell thickness has been investigated in the sandwich cell on the basis of this alignment. That is, the apparently uniform-like molecular alignment is due to this small twisted state along the cell thickness.

Recently, we found that the strong laser beam could dramatically change not only the switching pattern but also the spatial molecular orientation in chiral tilted smectic LC systems without using any photosensitive moiety. It just depends whether or not the aligning material has an absorption band in the wavelength region of the laser beam. In this letter we investigate the photocontrolled spatial molecular alignment in a thin sandwich cell which exhibits V-shaped switching.

A three-component Inui mixture (or Tokyo mixture) was investigated. ${ }^{2-4,8}$ We used two polyimides, RN1199 and RN1266 (Nissan Chemical Industries, Ltd.), as the aligning material. They were spin coated on glass substrates with an ITO coating. For a sandwich cell, one of the two substrates was rubbed unidirectionally with a rotating roller fitted with a velvet cloth. The cell gap was controlled with a ball spacer of $2 \mu \mathrm{m}$ diameter mixed with an UV curable adhesive. The
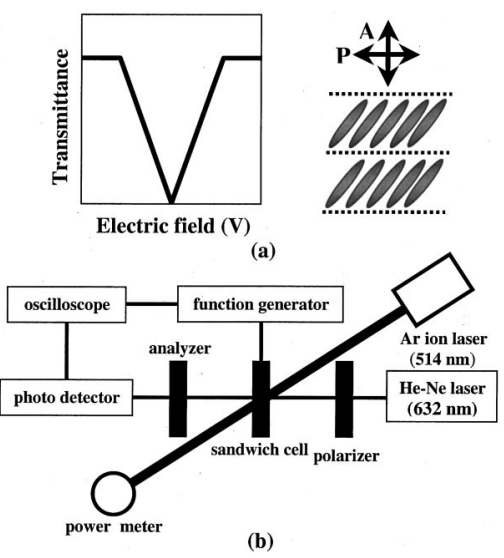

FIG. 1. (a) V-shaped switching pattern. (b) Schematic of the set up. 


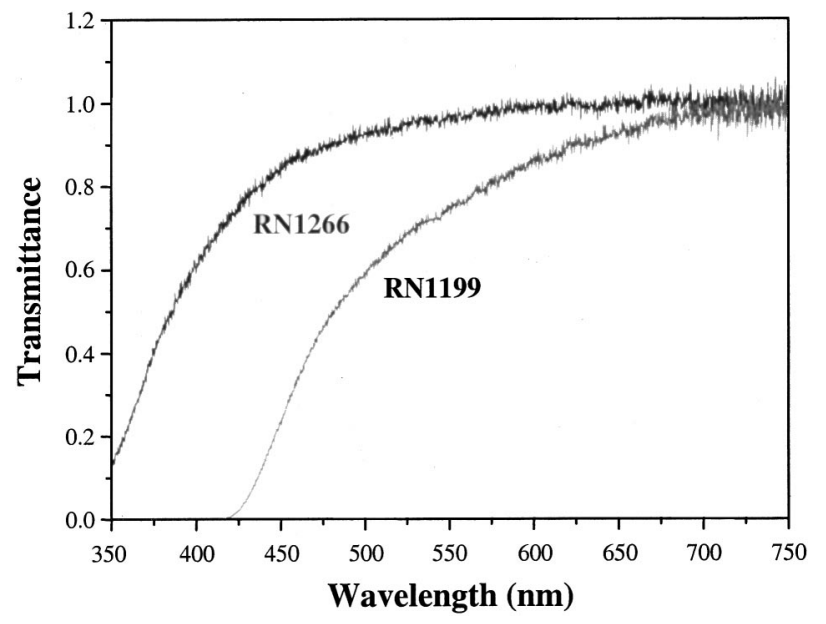

FIG. 2. Transmission spectra for the two polyimides.

switching behavior was probed using monochromatic light from a $\mathrm{He}-\mathrm{Ne}$ laser $(632 \mathrm{~nm})$, with a $2 \mathrm{~mm}$ aperture under crossed polarizers [Fig. 1(b)]. An Ar ion laser beam (514 nm) with a $3 \mathrm{~mm}$ aperture was irradiated in the cell during the switching process. The switching behavior was examined in $\mathrm{Sm} X^{*}$ phase $\left(50^{\circ} \mathrm{C}\right)$ under an electric field of triangular wave form with frequency of $1 \mathrm{~Hz}$ [the phase sequence: $\mathrm{AF}$ $\left(20-43^{\circ} \mathrm{C}\right.$ : coexistence with $\left.\mathrm{Sm} X^{*}\right) \mathrm{Sm} X^{*}\left(64^{\circ} \mathrm{C}\right) \mathrm{Sm} A$ $\left(68.5^{\circ} \mathrm{C}\right)$ Iso.$^{3}$

The two aligning materials, RN1199 and RN1266, show the different wavelength dependencies of transmittance given in Fig. 2; RN1199 has transmittance $T=0.64$ for $\lambda$ $=514 \mathrm{~nm}$, while RN1266 is almost transparent $(T=0.97)$ for the same wavelength. The light absorption in the RN1199 aligning material causes the switching pattern and the spatial molecular orientation to be different before and after the irradiation. Figure 3 shows the switching behavior observed with a $\mathrm{He}-\mathrm{Ne}$ laser during irradiation by an $\mathrm{Ar}$ ion laser. The cell used here was coated with RN1199. Before irradiation, the initial switching pattern shows the $\mathrm{V}$ shape with no hysteresis. Arrow 1 in Fig. 3(a) indicates the dark level when an applied field was turned off before and after switching. The dark level is almost the same in both cases and shows good extinction. However, the irradiation by the Ar laser beam induces hysteresis in the switching pattern. The hysteresis increases with increasing intensity of the laser beam and finally saturates at laser power of about $45 \mathrm{~mW}\left(0.64 \mathrm{~W} / \mathrm{cm}^{2}\right)$. For each switching pattern, however, the level of the transmittance at the tip of the V-shaped switching is almost the same. This shows that the molecular motion itself during switching has not changed in spite of the emergence of hysteresis. When the laser beam is turned off, the switching pattern reverts back to approximately its initial shape but with a little hysteresis [Fig. 3(e)].

Meanwhile, the long-term irradiation of the laser beam produces a different result. The laser power irradiation was fixed at $95 \mathrm{~mW}\left(1.34 \mathrm{~W} / \mathrm{cm}^{2}\right)$. Under this condition, the switching pattern maintains the same hysteresis independent of the irradiation time [Fig. 3(d)]. However, when the laser beam is turned off after $2 \mathrm{~h}$, the larger hysteresis persists compared to irradiation for a shorter time [Figs. 3(e) and 3(f)]. Note that the dark level [arrow 2 in Fig. 3(f)] rises

The texture (Fig. 5) with the extinction position parallel
when the field is turned off.
Downloaded 01 Jul 2010 to 134.226.1.229. Redistribution subject to AIP license or copyright; see http://apl.aip.org/apl/copyright.jsp

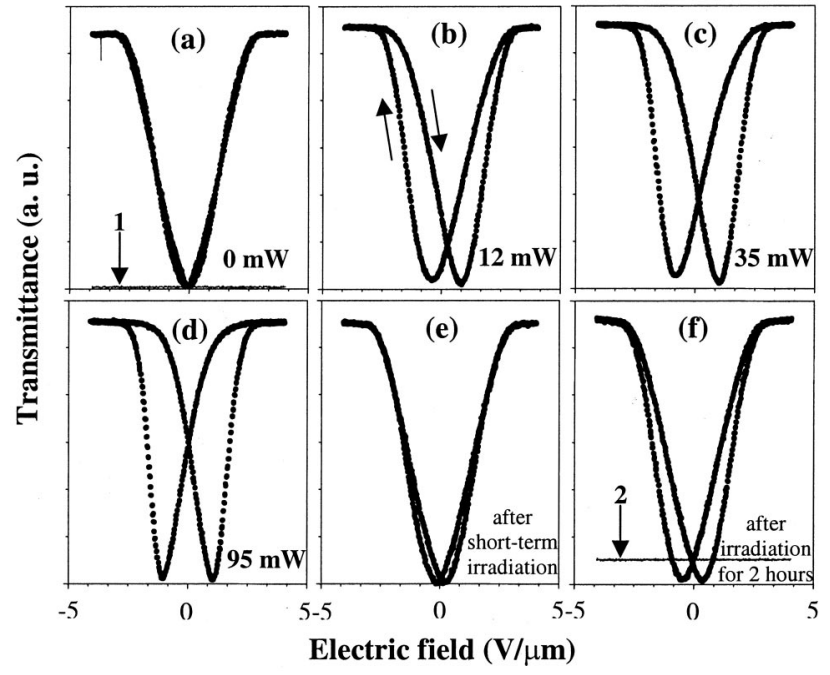

FIG. 3. Switching pattern affected by laser irradiation $(514 \mathrm{~nm})$ in the cell coated by polyimide RN1199.

The measurements show that the higher dark level in Fig. 3 after the long-term irradiation is due to a change in the spatial director $n$. Figure 4 shows the texture after irradiation with the Ar laser beam in $\mathrm{Sm} X^{*}$ phase $\left(50^{\circ} \mathrm{C}\right)$ for $2 \mathrm{~h}$. Before irradiation, the averaged optical axis is almost parallel to $l$ both inside and outside the circle. After irradiation, however, the averaged optical axis is found to be parallel to $r$ (inside the circle), i.e., the molecules lie along $r$. Of course, the maximum transmittance in this region is obtained when the stage is rotated by an angle of $45^{\circ}$ from $r$.

Irradiation with the Ar laser beam induces drastic changes not only in the switching pattern but also in the molecular orientation in $\operatorname{Sm} X^{*}$ phase. However, in $\operatorname{Sm} A$ phase, this phenomenon is not repeated. In addition, the irradiated light directly interacts with the aligning layer, not the liquid crystalline molecules, which is confirmed in the cell with RN1266 being almost transparent to the irradiated laser beam (Fig. 2). The V-shaped switching in such a cell (Fig. 5) is independent of the irradiation of the Ar laser beam. As a consequence, the texture remains unchanged after irradiation too. Meanwhile, the modified spatial molecular orientation seen in Fig. 4 returns to the original state through cooling after reheating to the isotropic phase. Accordingly, chemical damage of the aligning material by irradiation is ruled out.

The emergence of hysteresis during the irradiation by the Ar laser beam is due to an increase in the internal field $\left(E_{\text {int }}\right)$ applied to the liquid crystal layer. Before irradiation, in the field-induced uniform state all charges due to the alignment of $P$ are compensated for except residual charges on both surfaces that generate the depolarization field $\left(E_{\text {dep }}\right)$ in the cell. ${ }^{10}$ This $E_{\text {dep }}$ seems to be strongly shielded by the irradiation of the laser beam. So $E_{\text {int }}$ increases and hysteresis emerges. As a possible shielding mechanism of $E_{\text {dep }}$, the neutralization of the surface charges by the expediency of charge injection from the aligning layer ${ }^{10}$ can be considered. Figure 6 shows that $E_{\mathrm{LC}}=E_{\mathrm{ext}}-2 E_{\mathrm{PI}}$ and $E_{\mathrm{int}}=E_{\mathrm{LC}}-E_{\mathrm{dep}}$ where $E_{\text {ext }}$ is an applied external field and $E_{\mathrm{LC}}$ and $E_{\mathrm{PI}}$ are applied to the LC and the aligning layers, respectively.

The texture (Fig. 5) with the extinction position parallel 

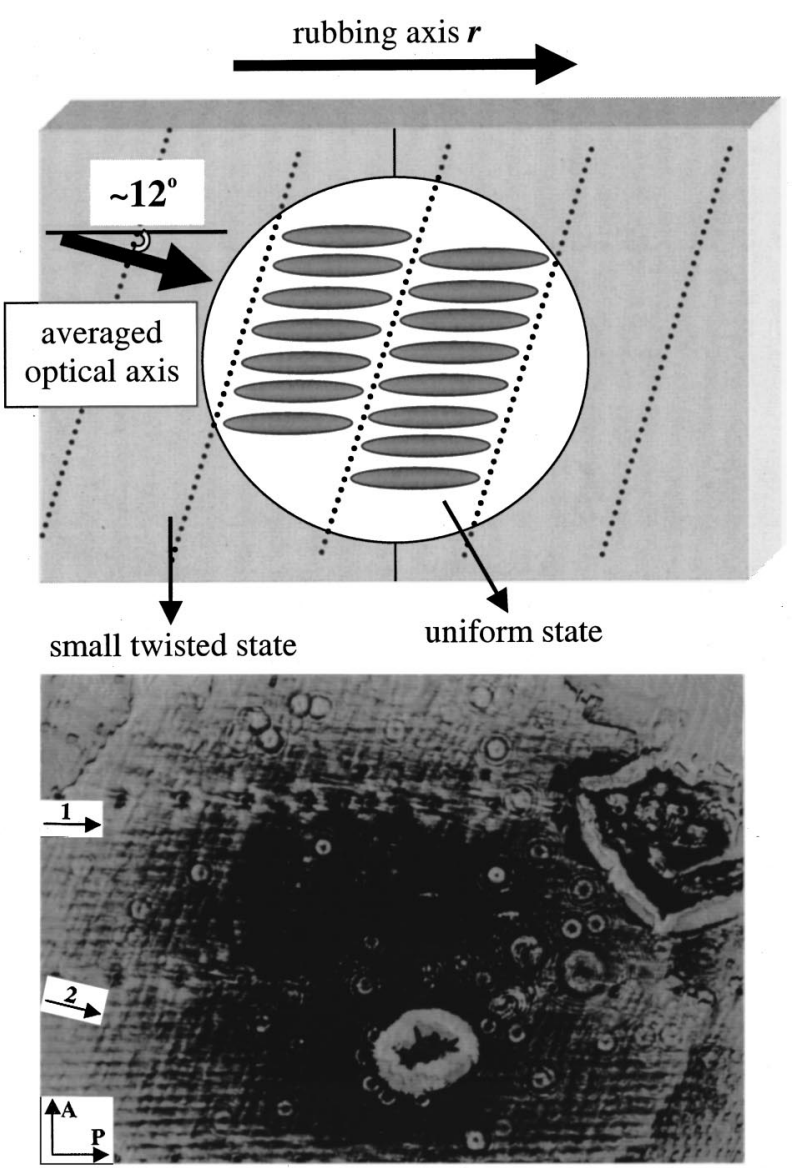

FIG. 4. Domain produced by irradiation by the Ar ion laser $(514 \mathrm{~nm})$ on a one-side-rubbed sandwich cell with aligning polyimide RN1199. The laser power is $95 \mathrm{~mW}\left(1.34 \mathrm{~W} / \mathrm{cm}^{2}\right)$, and the beam diameter is $3 \mathrm{~mm}$. Arrows 1 and 2 point to the rubbing axis $r$ and smectic layer normal $l$.

to $r$ is caused by a decrease in the polar interaction between the molecules and the unrubbed surface. In the $\operatorname{Sm} A-\operatorname{Sm} X^{*}$ transition, the molecules near the rubbed surface are rotated toward $r$ due to surface-molecule polar interactions. However, the strong in-plane anchoring by the rubbing treatment restrains all the molecules along $r$ below $2-3{ }^{\circ} \mathrm{C}$ from a $\operatorname{Sm} A-\operatorname{Sm} X^{*}$ phase transition. ${ }^{8}$ On the other hand, the molecules near the unrubbed surface tend to be rotated in the opposite direction with respect to $l$ due to polar anchoring. Finally the molecules are twisted along the cell thickness between such two different surface alignments. ${ }^{8}$ Neutralization of the surface polarization by irradiation extremely weakens the molecule-surface polar interaction. In this case,

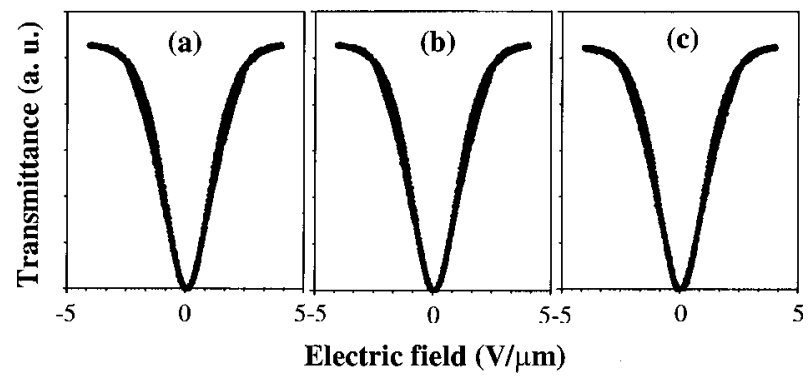

FIG. 5. V-shaped switching pattern in the cell coated by polyimide RN1266. (a) Before irradiation; (b) during irradiation; (c) after irradiation for $2 \mathrm{~h}$.

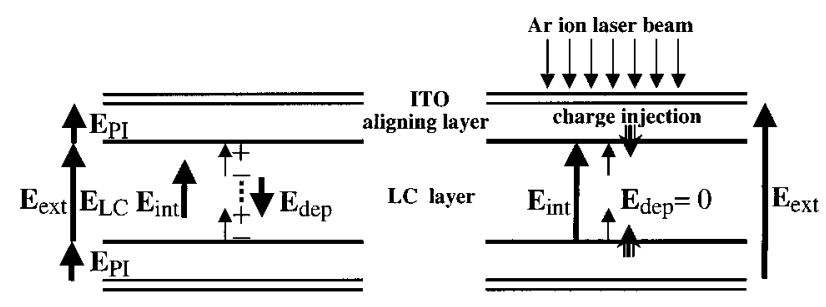

(a) before irradiation

(b) during irradiation

FIG. 6. Possible shielding mechanism of the depolarization field.

the molecular alignment near the rubbed surface is unaffected because the in-plane anchoring restrains the molecules. Meanwhile, the molecules near the unrubbed surface which are set free from polar anchoring are affected by the rubbed surface through intralayer molecular interaction. Consequently all the molecules are uniformly aligned parallel to $r$.

In-plane anchoring was investigated in the ferroelectric liquid crystals (FLCs) by Panarin et al. ${ }^{11}$ The total surface energy on a one-side-rubbed surface is given as $W_{t}=W_{s}$ $+W_{0} . W_{s}$ involves polar and nonpolar anchoring strengths and $W_{0}$ is the in-plane anchoring term due to the rubbing treatment. $^{11,12}$ The surface molecular alignment parallel to $r$ must be a result of the predominant in-plane anchoring $W_{0}$.

In summary, the light absorption of the aligning polyamide expedites charge injection into the LC layer and causes neutralization of the surface polarization and shielding of the depolarization field. Consequently, a large hysteresis from the $\mathrm{V}$ shape emerges during the switching and a different spatial molecular orientation is observed due to a change in the surface-molecule interactions before and after the longterm irradiation by the Ar laser beam. Photocontrolled molecular orientation indicates that both the sandwich and the open cell have the same molecular alignment on the rubbed surface. Hence, the apparently uniform-like molecular orientation is due to in-plane anchoring and its real spatial structure is a small twisted state along the cell thickness. ${ }^{8}$

This work was funded by the HEA under the initiative of Advanced Materials. The authors thank Professor J. Hegarty for allowing them to use his Laser Physics Laboratory. Nissan Chemical Industries are thanked for aligning polymides.

${ }^{1}$ A. Fukuda, Proceedings of Asia Display'95 (Institute Television Engineering, Japan, 1995), p. 61.

${ }^{2}$ S. Inui, N. Iimura, T. Suzuki, H. Iwane, M. Miyachi, Y. Takanishi, and A. Fukuda, J. Mater. Chem. 6, 671 (1996)

${ }^{3}$ S. S. Seomun, Y. Takanishi, K. Ishikawa, H. Takezoe, and A. Fukuda, Jpn. J. Appl. Phys., Part 2 37, L691 (1998).

${ }^{4}$ T. Matsumoto et al., J. Mater. Chem. 9, 2015 (1999).

${ }^{5}$ B. C. Park, S. S. Seomun, M. Nakata, Y. Takanishi, K. Ishikawa, H. Takezoe, and A. Fukuda, Jpn. J. Appl. Phys., Part 1 38, 1474 (1999).

${ }^{6}$ P. Rudquist et al., J. Mater. Chem. 9, 1257 (1999).

${ }^{7}$ N. A. Clark, D. Coleman, and J. E. Maclennan, Liq. Cryst. 27, 985 (2000).

${ }^{8}$ S. S. Seomun, T. Fukuda, A. Fukuda, J.-G. Yoo, Yu. P. Panarin, and J. K. Vij, J. Mater. Chem. 10, 2791 (2000)

${ }^{9}$ T. Yoshida, T. Tanaka, J. Ogura, H. Wakai, and H. Aoki, SID'97 Digest 841 (1997).

${ }^{10}$ K. H. Yang, T. C. Chieu, and S. Osofsky, Appl. Phys. Lett. 55, 125 (1989).

${ }^{11}$ Yu. P. Panarin, S. T. MacLughadha, and J. K. Vij, Phys. Rev. E 52, R17 (1995).

${ }^{12}$ M. A. Handschy, N. A. Clark, and S. T. Lagerwall, Phys. Rev. Lett. 51, 471 (1983). 